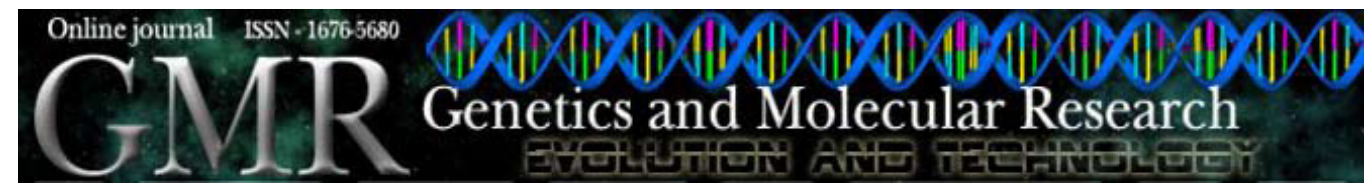

Short Communication

\title{
Citrullinemia type I: molecular screening of the ASS1 gene by exonic sequencing and targeted mutation analysis
}

\author{
R. Marquis-Nicholson ${ }^{1}$, E. Glamuzina ${ }^{2}$, D. Prosser ${ }^{1}$, C. Wilson ${ }^{2}$ and D.R. Love ${ }^{1,3}$ \\ ${ }^{1}$ Diagnostic Genetics, LabPLUS, Auckland City Hospital, \\ Auckland, New Zealand \\ 2Paediatric Metabolic Service, Starship Children's Hospital, Auckland, New Zealand \\ ${ }^{3}$ School of Biological Sciences, University of Auckland, Auckland, New Zealand
}

Corresponding author: D.R. Love

E-mail: donald1@adhb.govt.nz

Genet. Mol. Res. 9 (3): 1483-1489 (2010)

Received April 26, 2010

Accepted May 21, 2010

Published August 3, 2010

DOI 10.4238/vol9-3gmr834

\begin{abstract}
We developed a mutation-screening protocol for the ASS1 gene in order to guide clinical management of neonates with elevated citrulline detected during routine newborn screening. An exon-based amplification and sequencing method was designed and successfully applied to patients to identify disease-associated mutations. The sequencing-based method was applied to three patients with mild or asymptomatic clinical courses. Identification of a homozygous mutation in these patients, c. $787 \mathrm{G}>\mathrm{A}$ (p.Val263Met), led to the development of a tetra-primer ARMS-PCR method that successfully detected the mutation in DNA extracted from blood or from Guthrie card spots.
\end{abstract}

Key words: ASS1 gene; Tetra-primer ARMS-PCR; Sequencing; Citrullinemia type I 


\section{INTRODUCTION}

Citrullinemia type I is a rare urea-cycle disorder caused by a deficiency of argininosuccinate synthetase (Häberle et al., 2003). This cytosolic enzyme catalyzes the conversion of citrulline and aspartate to argininosuccinate, primarily in periportal hepatocytes, but also in other body tissues involved in the synthesis of arginine (Häberle et al., 2003; Engel et al., 2009). The disorder is inherited in an autosomal recessive manner, and is due to a heterogeneous group of mutations in the ASS1 gene on chromosome 9 (Häberle et al., 2002; Sander et al., 2003; Engel et al., 2009).

In classical citrullinemia type I, failure of the urea cycle leads to the accumulation of citrulline, glutamine and ammonia in the serum and increased excretion of orotic acid in the urine (Häberle et al., 2002, 2003; Engel et al., 2009). Individuals with classical citrullinemia type I often present early in the neonatal period with metabolic coma due to hyperammonemia, which if untreated proves to be fatal (Sander et al., 2003; Häberle et al., 2003), but with treatment instead leads to developmental delay of varying degrees of severity (Häberle et al., 2002). Late onset forms of citrullinemia can also occur (Häberle et al., 2003), with metabolic decompensation triggered by increased catabolic stress, as in illness or the post-partum period (Dimmock et al., 2008). Mild or asymptomatic forms of the disease, in which there is elevated serum citrulline but no hyperammonemia, have also been described (Häberle et al., 2002, 2003; Sander et al., 2003). Treatment, when required, consists of dietary protein restriction, substitution of arginine and other essential amino acids, and the use of medication to allow the alternative detoxification of surplus nitrogen (Engel et al., 2009). The frequency with which mild cases of citrullinemia type I are diagnosed has increased dramatically over the last 5-10 years as more countries offer extended newborn metabolic screening programs (E-NBS) (Häberle et al., 2003).

The clinical course and prognosis of citrullinemia type I are difficult to predict by biochemical means alone. The level of citrulline in the serum cannot be used to determine the severity of the disease, although an E-NBS cut-off of $1000 \mu \mathrm{M}$ has been proposed, as classical citrullinemia type I is almost always associated with levels higher than this (Engel et al., 2009). Since the discovery of the pivotal role of the ASS1 gene and the elucidation of its structure, it has been possible to perform molecular genetic analysis of affected individuals to determine the specific mutations underlying the disease (Engel et al., 2009; Häberle et al., 2003). There are now a total of 87 mutations in the ASS1 gene that are known to cause type I citrullinemia, and this number continues to rise as sequence analysis is carried out on an increasing number of patients.

A predictable problem with E-NBS, however, is the identification of biochemical abnormalities in an otherwise well baby (Waisbren et al., 2003). These abnormalities are often less extreme than those found in individuals with the classical form of the particular metabolic disorder. As a consequence, predicting the character of a patient's long-term clinical course and determining whether to initiate treatment remain a challenge (Häberle et al., 2003; Sander et al., 2003; Waisbren et al., 2003; Dimmock et al., 2008). In the context of citrullinemia type I, it is important to weigh the necessity of commencing appropriate treatment early against the need to avoid unnecessary treatment in the case of clinically insignificant citrulline elevation.

As a means of supporting E-NBS, and to help guide the clinical management of babies with mildly elevated citrulline, the decision was made to develop a mutation screening proto- 
col for the ASS1 gene. The study presented here describes this protocol, together with the development of a mutation-targeted approach that can be applied to either blood or Guthrie cards.

\section{MATERIAL AND METHODS}

\section{Primer design and optimization}

Primers were designed to flank each of the 14 coding exons (3-16) of the ASSI gene, including $50 \mathrm{bp}$ of the flanking intronic regions. The mRNA sequence of interest was identified through the public UCSC genome browser (http://genome.ucsc.edu). This website provides a direct link to ExonPrimer for the design of primers flanking coding exons. All primers were checked for single-nucleotide polymorphisms using the software tool available from the National Genetic Reference Laboratory, Manchester (http://ngrl.man.ac.uk/ SNPCheck/SNPCheck.html). The primers were tailed with M13 sequences (Table 1) and were synthesized by Invitrogen Ltd. For comparative analysis, an alternative set of primers published by Häberle et al. (2002) was also used to amplify the coding exons of the ASS1 gene.

\begin{tabular}{|c|c|c|}
\hline Exon & Primer & Sequence \\
\hline \multirow[t]{2}{*}{3} & Forward & TGTAAAACGACGGCCAGTGGCACTGGCTGTCTCAGG \\
\hline & Reverse & CAGGAAACAGCTATGACCGACAGGACAGGTTGCAGGAC \\
\hline \multirow[t]{2}{*}{4} & Forward & TGTAAAACGACGGCCAGTATGGTGTGAACTCAGGGCTC \\
\hline & Reverse & CAGGAAACAGCTATGACCAGGAGCATCCACCACTGC \\
\hline \multirow[t]{2}{*}{5} & Forward & TGTAAAACGACGGCCAGTGGGCTCTGTATGCCAGATG \\
\hline & Reverse & CAGGAAACAGCTATGACCGAGGGGTGCTCATCTCCTTC \\
\hline \multirow[t]{2}{*}{6} & Forward & TGTAAAACGACGGCCAGTTTGTCCTCACGTCCTCCC \\
\hline & Reverse & CAGGAAACAGCTATGACCCCCTCCTCCCCATCAGG \\
\hline \multirow[t]{2}{*}{7} & Forward & TGTAAAACGACGGCCAGTCCAGCTCTGCAGCTTACAGG \\
\hline & Reverse & CAGGAAACAGCTATGACCCCATCACATGGGGTCAGTC \\
\hline \multirow[t]{2}{*}{8} & Forward & TGTAAAACGACGGCCAGTCCTGGGACGGACCTCAC \\
\hline & Reverse & CAGGAAACAGCTATGACCCGTCCTTTGGAATGAGCC \\
\hline \multirow[t]{2}{*}{9} & Forward & TGTAAAACGACGGCCAGTACAATGGGGTGTGTGTGTTG \\
\hline & Reverse & CAGGAAACAGCTATGACCTGGAGCTGCTACCACCAAAG \\
\hline \multirow[t]{2}{*}{10} & Forward & TGTAAAACGACGGCCAGTGGGGAAATGGACAGAGGAG \\
\hline & Reverse & CAGGAAACAGCTATGACCCCTAGGTTCCCAGGCAGG \\
\hline \multirow[t]{2}{*}{11} & Forward & TGTAAAACGACGGCCAGTAGACTCCTCCGCTGAGCC \\
\hline & Reverse & CAGGAAACAGCTATGACCCACAACCATTAGCTGCAACG \\
\hline \multirow[t]{2}{*}{12} & Forward & TGTAAAACGACGGCCAGTGTGGGTGACTCTGAGCCTTG \\
\hline & Reverse & CAGGAAACAGCTATGACCCTCAGGGATCTCTGGGTTTG \\
\hline \multirow[t]{2}{*}{13} & Forward & TGTAAAACGACGGCCAGTTCATTTGCTGACAGTTTGGG \\
\hline & Reverse & CAGGAAACAGCTATGACCACTTTGGGATCCCTTGTGAG \\
\hline \multirow[t]{2}{*}{14} & Forward & TGTAAAACGACGGCCAGTCCTGTGTCCTCGCGGTG \\
\hline & Reverse & CAGGAAACAGCTATGACCCAGGCACAGATGTCTTGAGG \\
\hline \multirow[t]{2}{*}{$15^{*}$} & Forward & TGTAAAACGACGGCCAGTCTAGGGCTCTCCAACCTTTGGGTG \\
\hline & Reverse & CAGGAAACAGCTATGACCTTACACCTGGGGGCTCTCAA \\
\hline \multirow{2}{*}{16} & Forward & TGTAAAACGACGGCCAGTGAACCCAGTGTGTGTTGTTATTG \\
\hline & Reverse & CAGGAAACAGCTATGACCAATTAGCGCCTGTACTTGGG \\
\hline
\end{tabular}

Primers for all exons were designed using ExonPrimer (accessed through UCSC, http://genome.ucsc.edu $<$ http://genome. ucsc.edu/ $>$ ), with the exception of exon 15 (indicated by asterisk), which was designed using the software program FastPCR (http://www.biocenter.helsinki.fi/bi/Programs/fastpcr.htm). The sequences shown in blue and red correspond to 18-mer M13 bacteriophage sequences that were added to the 5' end of all forward and reverse primers, respectively.

\section{PCR amplification and DNA sequencing}

DNA was extracted from peripheral blood-EDTA samples using the Gentra Puregene 
DNA Extraction kit (Qiagen). Polymerase chain reaction (PCR) was performed using 1 U Faststart Taq DNA polymerase (Invitrogen Ltd.), $50 \mathrm{ng}$ genomic DNA, $2 \mathrm{mM} \mathrm{MgCl}, 0.8 \mu \mathrm{M}$ forward and reverse primers, with the following cycle conditions: $95^{\circ} \mathrm{C}$ for $4 \mathrm{~min}, 35$ cycles of $94^{\circ} \mathrm{C}$ for 45 $\mathrm{s}, 60^{\circ} \mathrm{C}$ for $30 \mathrm{~s}, 72^{\circ} \mathrm{C}$ for $30 \mathrm{~s}$, and then a final extension at $72^{\circ} \mathrm{C}$ for $10 \mathrm{~min}$. In the case of exon 15, the ExonPrimer-designed and previously designed primers (Häberle et al., 2002) failed to produce amplicons. As a consequence, an alternative primer design program (FastPCR; http:// www.biocenter.helsinki.fi/bi/Programs/fastpcr.htm) was used to design a number of primer pairs flanking this exon, one of which was used routinely for the amplification of exon 15.

Each PCR mixture $(5 \mu \mathrm{L})$ was treated with ExoSAP-IT (Affymetrix) prior to bidirectional DNA sequencing using M13 forward and reverse primers and Big-Dye Terminator v3.0 (Applied Biosystems Ltd.). A volume of $20 \mu \mathrm{L}$ of sequenced product was purified using an automated Clean-Seq (Agencourt) procedure with the aid of an epMOTION 5075 liquid handling robot (Eppendorf). Fifteen microliters of purified product was then subjected to capillary electrophoresis using an Applied Biosystems model 3130xls Genetic Analyser.

The analysis of trace sequences was performed using Variant Reporter v1.0 (Applied Biosystems). Genebank NM_000050.4 was used as the reference sequence, with cDNA number +1 corresponding to the $\bar{A}$ of the translation initiation codon (codon 1 ). The quality control values for the sequence traces obtained using the ExonPrimer/FastPCR primers were consistently higher than previously designed primers (Häberle et al., 2002). As a result, the ExonPrimer/Fast PCR primers were preferentially selected for the final set of primers.

\section{Tetra-primer ARMS-PCR}

Tetra-primer ARMS (amplification refractory mutation system)-PCR has been reported to be a simple and economical point mutation detection method (Ye et al., 2001; Okayama et al., 2004). This approach uses two primer pairs (inner and outer) to amplify two different potential alleles in a single PCR. We used the freely accessible online primer design program (available at http://cedar.genetics.soton.ac.uk) to design primers suitable for applying the technique to the target DNA sequence in Exon 12 of the ASS1 gene. Genomic DNA was extracted from peripheral blood-EDTA samples using the Gentra Puregene DNA Extraction kit (Qiagen) or the QIAmp DNA Miniblood kit (Qiagen) in the case of Guthrie cards. DNA was amplified using the conditions described above but with $0.4 \mu \mathrm{M}$ of each of the four primers. Gel electrophoresis of the PCR products was performed and the genotype determined.

\section{RESULTS}

Our initial attempts at the amplification of all coding exons of the ASSI gene using previously designed primers (Häberle et al., 2002) were not generally successful. We, therefore, developed a streamlined pre-analytical process for primer design that we applied to the ASSI gene, which has since been translated to all other genes that we sequence in a small diagnostic laboratory (Doherty E, Love J, Marquis-Nicholson R, Prosser D and Love DR, unpublished results). All coding sequence exons of the ASS1 gene were successfully amplified using a single PCR condition, and the double-stranded sequence quality was consistently high as determined using the quality metrics of the Variant Reporter Software (Applied Biosystems Ltd.).

We undertook the analysis of three patients who had elevated citrulline levels compatible 
with a clinical diagnosis of citrullinemia. Interestingly, all three patients were homozygous for the same mutation in exon 12 of the ASS1 gene: c.787G>A, p.Val263Met (Figure 1), which has previously been reported in patients of predominantly Turkish origin (Häberle et al., 2003; Sander et al., 2003; Engel et al., 2009).

A

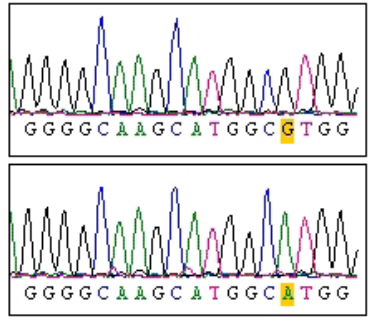

Figure 1. Partial exon 12 sequence encompassing the c.787G $>$ A (p.V263M) mutation. Electropherograms begin at the first nucleotide of exon $12(\mathrm{G})$. The c. $787 \mathrm{G}>$ A mutation is highlighted in yellow. A. Negative control (homozygous G; wild-type sequence). B. Patient 1 (homozygous A).

Given the success of the above sequence approach, we decided to develop a targeted mutation detection approach. We used the online tetra-primer ARMS-PCR software package to design primers to detect the c.787G $>$ A (p.Val263Met) mutation (Figure 2).

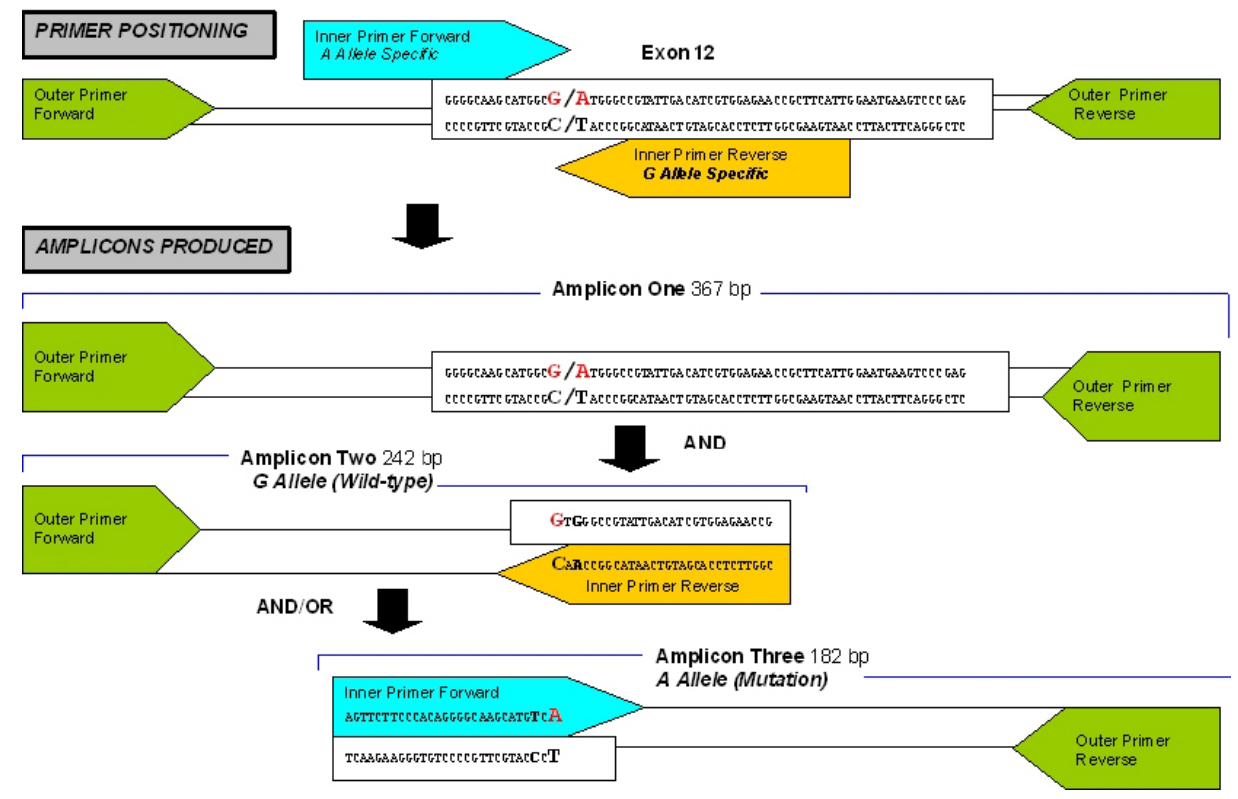

Figure 2. Diagrammatic representation of the tetra-primer ARMS-PCR method. Four primers are designed: two outer primers and two inner allele-specific primers that are targeted to the c.787G $>$ A mutation (p.V263M; highlighted in bold red in exonic sequence) in the ASS1 gene. Allele-specific primers are shown in blue (forward) and yellow (reverse), with mismatched bases at position -2 from the 3 ' terminal bases highlighted in bold. 
PCR was performed under a range of conditions during the optimization process, and the appropriate inner:outer primer ratio and reaction conditions were determined. Tetra-primer ARMS-PCR was able to detect the mutation using DNA extracted from either blood or from Guthrie cards (Figure 3).

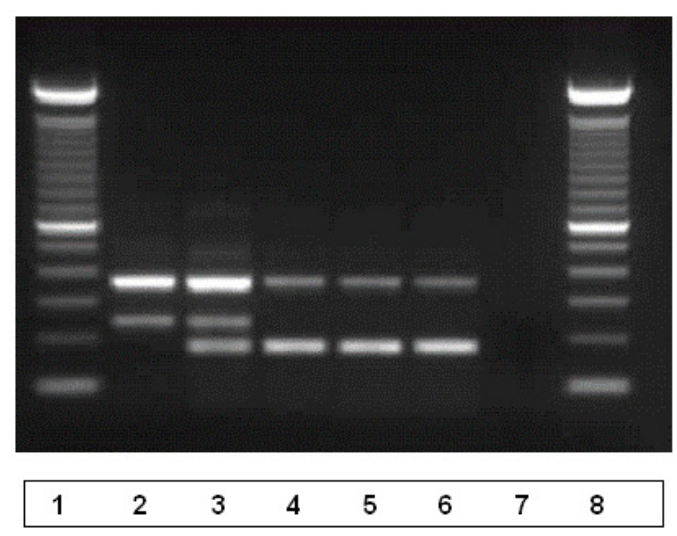

Figure 3. Agarose gel electrophoresis of tetra-primer ARMS-PCR amplification. Lanes 1 and $8=100$-bp ladder (Invitrogen Ltd.); lane 2 = negative control (homozygous G); lane 3 = heterozygote (mixture of DNAs from negative control and patient 1); lanes 4-6= patients 1-3; lane 7 = no DNA control.

\section{DISCUSSION}

There are 10 to 14 homologous copies of the ASS gene spread throughout the human genome, but the functionally relevant copy, $A S S 1$, is located in the long arm of chromosome 9 (Engel et al., 2009). A wide range of mutations have been implicated in citrullinemia type I (Häberle et al., 2002; Engel et al., 2009). This heterogeneous group involves all exons, except exons 1 and 2, which encode the 5' untranslated region of the gene transcript, and exon 16 (Engel et al., 2009). As yet there is no definite genotype-phenotype correlation (Dimmock et al., 2008), but increasing use of molecular analysis has led to the establishment of firmer links between specific mutations and particular clinical courses - e.g., mild or severe, requiring treatment or not requiring treatment. This correlation is complicated, however, by compound heterozygosity. Of the twenty mutations detected in mild disease to date, only four have been seen in a homozygous state (Engel et al., 2009). One of these is the c.787G $>$ A (p.Val263Met) mutation found in the patients in this study.

The study reported here is of relevance as it describes a sequence-based approach that is robust and provides high-quality data for mutation screening. In addition, the targeted mutation approach using the tetra-primer ARMS-PCR strategy is simple to implement, offers a fast turnaround time, and can be applied to very small sample volumes and sample types. It is our view that the use of molecular approaches can assist in the confirmation of an apparently benign clinical course, and that the entire coding sequence of the ASS1 gene can be captured, or more targeted strategies can be used to identify mutations that may be more common in defined population groups. 


\section{ACKNOWLEDGMENTS}

Research supported by the Ministry of Health of the New Zealand Government for the development of ASS1 gene mutation analysis in the Newborn Metabolic Screening Programme.

\section{REFERENCES}

Dimmock DP, Trapane P, Feigenbaum A, Keegan CE, et al. (2008). The role of molecular testing and enzyme analysis in the management of hypomorphic citrullinemia. Am. J. Med. Genet. A 146A: 2885-2890.

Engel K, Hohne W and Haberle J (2009). Mutations and polymorphisms in the human argininosuccinate synthetase (ASS1) gene. Hum. Mutat. 30: 300-307.

Häberle J, Pauli S, Linnebank M, Kleijer WJ, et al. (2002). Structure of the human argininosuccinate synthetase gene and an improved system for molecular diagnostics in patients with classical and mild citrullinemia. Hum. Genet. 110: 327-333.

Häberle J, Pauli S, Schmidt E, Schulze-Eilfing B, et al. (2003). Mild citrullinemia in Caucasians is an allelic variant of argininosuccinate synthetase deficiency (citrullinemia type 1). Mol. Genet. Metab. 80: 302-306.

Okayama N, Fujimura K, Nakamura J, Suehiro Y, et al. (2004). Evaluation of a new efficient procedure for singlenucleotide polymorphism genotyping: tetra-primer amplification refractory mutation system-polymerase chain reaction. Clin. Chem. Lab. Med. 42: 13-16.

Sander J, Janzen N, Sander S, Steuerwald U, et al. (2003). Neonatal screening for citrullinaemia. Eur. J. Pediatr. 162: 417-420.

Waisbren SE, Albers S, Amato S, Ampola M, et al. (2003). Effect of expanded newborn screening for biochemical genetic disorders on child outcomes and parental stress. JAMA 290: 2564-2572.

Ye S, Dhillon S, Ke X, Collins AR, et al. (2001). An efficient procedure for genotyping single nucleotide polymorphisms. Nucleic Acids Res. 29: E88. 